Bull. Soc. math. France

131 (2), 2003, p. 267-287

\title{
HYPERBOLIC SYSTEMS ON NILPOTENT COVERS
}

\author{
By Yves Coudene
}

\begin{abstract}
We study the ergodicity of the weak and strong stable foliations of hyperbolic systems on nilpotent covers. Subshifts of finite type and geodesic flows on negatively curved manifolds are also considered.

RÉSUMÉ (Systèmes hyperboliques sur des revêtements nilpotents)

Nous étudions les propriétés ergodiques des feuilletages stables forts et faibles des systèmes hyperboliques définis sur un revêtement nilpotent. Les chaînes de Markov et les flots géodésiques en courbure négative sont aussi étudiés.
\end{abstract}

\section{Introduction}

This article is devoted to the study of the ergodicity of the stable foliation of an hyperbolic flow defined on a nilpotent cover of a manifold.

The geodesic flow on a compact two dimensional manifold of constant negative curvature is one of the simplest example of hyperbolic flow. E. Hopf [19], G.A. Hedlund [17], [18] showed the ergodicity of the associated horospheric flow with respect to the Liouville measure. H. Furstenberg obtained its unique ergodicity [15]. This was generalized to the case of the stable foliation of an

Texte reçu le 24 janvier 2002, révisé le 28 mai 2002, accepté le 28 mai 2002.

Yves Coudene, IRMAR, Université Rennes 1, Campus de Beaulieu, bât. 22, 35042 Rennes Cedex (France) • E-mail : yves.coudene@univ-rennes1.fr 2000 Mathematics Subject Classification. — 37D10, 37D20, 37D40.

Key words and phrases. - Covering space, ergodic theory, geodesic flow, hyperbolic flow, invariant manifolds, Markov chain. 
hyperbolic flow by D.V. Anosov [2] and R. Bowen and B. Marcus [6]. In the context of infinite measure ergodic theory, it was natural to ask whether ergodicity of the foliation is again true on a regular cover.

This question has been investigated by many authors in the last decade. M. Babillot, F. Ledrappier studied the case of the geodesic flow on a compact manifold of constant negative curvature [3]. The extension of the flow to an abelian cover admits a number of invariant measures; they were able to show that the stable foliation of the extension is ergodic with respect to these measures. Their proof relies on counting estimates obtained by transfer operator technics. Using results from harmonic analysis, V. Kaimanovich was able to generalize their result to nilpotent extensions for the Liouville measure [20]. In another direction, M. Pollicott considered homologically full Anosov flows and obtained ergodicity of the foliation on an abelian cover for the measure of maximal entropy [23]. The method makes use of a symbolic model. We studied the case of Axiom A flows [10]; for these systems, the stable foliation may be non ergodic and we gave necessary and sufficient conditions in term of periodic orbits in order to get that ergodicity. Very few hypothesis were made on the measure, in order to treat the case of the Liouville measure, measure of maximal entropy and harmonic measure. Following M. Pollicott, we were working in a symbolic setting, so that two restrictive hypothesis were needed: the non-wandering set of the flow on the basis of the cover had to be compact and the cover was abelian. R. Solomyak [27], U. Hamenstadt [16] and then J. Aaronson, R. Solomyak and O. Sarig [1], also obtained several results, using methods of independent interest.

The goal of this article is to provide an unified approach to these problems and to recover these results using just the hyperbolicity of the systems. No compactness assumption on the wandering set is made and the cover can be taken nilpotent. As a consequence, we obtain a number of theorems, conjectured from the beginning, but which resisted all previous attempts, like the finite volume case.

We first state a general result concerning the ergodicity of the stable foliation of systems admitting local product structures. The second part of the article is devoted to the proof of that result. It is then applied to hyperbolic flows, subshifts of finite type and geodesic flows on negatively curved manifolds.

\section{Main result}

Let $G$ be a Polish group acting continuously on a Polish space $\widehat{X}$ and $\widehat{\phi}_{t}$ a continuous flow on $\widehat{X}$ commuting with the $G$ action. The quotient of $\widehat{X}$ by $G$ is denoted by $X$, the projection from $\widehat{X}$ to $X$ by $\pi$, and the quotient flow on $X$ by $\phi_{t}$. Let us assume that the quotient topology on $X$ is given by a metric for which $\pi$ is Lipschitz. Strong stable sets are defined by:

TOME $131-2003-\mathrm{N}^{\mathrm{O}} 2$ 


$$
\begin{aligned}
& W^{s s}(x):=\left\{y \in X \mid \lim _{t \rightarrow \infty} d\left(\phi_{t}(x), \phi_{t}(y)\right)=0\right\} ; \\
& W_{\varepsilon}^{s s}(x):=\left\{y \in W^{s s}(x) \mid d\left(\phi_{t}(x), \phi_{t}(y)\right) \leq \varepsilon \text { for all } t \geq 0\right\} ; \\
& \widehat{W}^{s s}(\widehat{x}):=\left\{\widehat{y} \in \widehat{X} \mid \lim _{t \rightarrow \infty} d\left(\widehat{\phi}_{t}(\widehat{x}), \widehat{\phi}_{t}(\widehat{y})\right)=0\right\} ; \\
& \widehat{W}_{\varepsilon}^{s s}(\widehat{x}):=\left\{\widehat{y} \in \widehat{W}^{s s}(\widehat{x}) \mid d\left(\widehat{\phi}_{t}(\widehat{x}), \widehat{\phi}_{t}(\widehat{y})\right) \leq \varepsilon \text { for all } t \geq 0\right\} .
\end{aligned}
$$

The weak stable sets $\widehat{W}^{w s}, W^{w s}$ of $\widehat{\phi}_{t}$ and $\phi_{t}$ are equal to $\widehat{\phi}_{\mathbb{R}}\left(\widehat{W}^{s s}\right)$ and $\phi_{\mathbb{R}}\left(W^{s s}\right)$. One also defines the strong unstable sets $\widehat{W}^{s u}, \widehat{W}_{\varepsilon}^{s u}, W^{s u}$ and $W_{\varepsilon}^{s u}$ of $\widehat{\phi}_{t}$ and $\phi_{t}$; these are the stable sets of $\widehat{\phi}_{-t}$ and $\phi_{-t}$.

Let $\tau$ be a periodic orbit of $\phi_{t}$ on $X$ and $\widehat{x} \in \widehat{X}$ a preimage of a point on the orbit $\tau$. The period of $\tau$ is denoted by $\ell(\tau)$. The Frobenius element $[\tau]$ in $G$ is the translation in the fiber along $\tau: \widehat{\phi}_{\ell(\tau)}(\widehat{x})=[\tau](\widehat{x})$. Its conjugacy class does not depend on the chosen preimage. If it belongs to the center of $G$, it is independant of the chosen preimage.

The next three uniformity assumptions are satisfied, for example, if the $G$ action on $\widehat{X}$ is isometric and the distance on $X$ is locally equivalent to the distance $d^{\prime}(x, y)=d_{\widehat{X}}\left(\pi^{-1} x, \pi^{-1} y\right)$.

- For all $g \in G$, the function $\widehat{x} \mapsto g \widehat{x}$ is uniformly continuous on $\widehat{X}$. In particular, this implies the relation $g \widehat{W}^{s s}(\widehat{x})=\widehat{W}^{s s}(g \widehat{x})$ for all $\widehat{x}$.

- For all $v \in X, \varepsilon>0$ and $T \geq 0$, there is a $\delta>0$ such that for all $\widehat{v} \in \pi^{-1}(v), d(\widehat{x}, \widehat{v})<\delta$ implies $d\left(\widehat{\phi}_{t}(\widehat{x}), \widehat{\phi}_{t}(\widehat{v})\right)<\varepsilon$ for all $|t| \leq T$.

- Let $v$ some point in $X$. If $\left\{\widehat{x}_{n}\right\}$ is a sequence in $\widehat{X}, d\left(\pi\left(\widehat{x}_{n}\right), v\right) \rightarrow 0$ implies $d\left(\widehat{x}_{n}, \pi^{-1} v\right) \rightarrow 0$.

The strong unstable foliation $\widehat{W}^{\text {su }}$ is said to be locally contracting uniformly in fibers if for any $v \in X$, there is an $\varepsilon>0$ such that for any $\delta_{0}>0$, one can find a $t_{0} \in \mathbb{R}$ so that $d\left(\widehat{\phi}_{-t}(\widehat{x}), \widehat{\phi}_{-t}(\widehat{y})\right) \leq \delta_{0}$ whenever $t \geq t_{0}, d\left(\widehat{x}, \pi^{-1} v\right)<\varepsilon$, $d\left(\widehat{y}, \pi^{-1} v\right)<\varepsilon$ and $y \in \widehat{W}_{\varepsilon}^{s u}(x)$.

The flow $\widehat{\phi}_{t}$ is said to admit a local product structure if, for all points $v \in X$, and all $\varepsilon>0$, there exists an $\varepsilon_{0}>0$, a neighborhood $V$ of $\pi^{-1} v$ with $\varepsilon_{0}<d\left(\pi^{-1} v, V^{c}\right), d\left(x, \pi^{-1} v\right)<\varepsilon$ for all $x \in V$, and positive constants $\delta_{1}, \delta_{2}$ less than $\varepsilon$, such that for all $x, y \in V$ with $d(x, y) \leq \delta_{1}$, there is a point $\langle x, y\rangle \in V$, a real number $t$ with $|t| \leq \delta_{2}$, and an element $g \in G$ with $d(g$, id $) \leq \delta_{2}$, so that:

$$
\widehat{W}_{\delta_{2}}^{s s}\left(g \widehat{\phi}_{t}(x)\right) \cap \widehat{W}_{\delta_{2}}^{s u}(y)=\langle x, y\rangle
$$

The maps sending $(x, y)$ to $\langle x, y\rangle, g$ and $t$ are furthermore supposed to be Borel maps; in practice, they will even be continuous on $V$.

The spaces $\widehat{X}$ and $X$ are endowed with two Borel measures, $\widehat{\mu}$ and $\mu$, in such a way that the projection $\pi: \widehat{X} \rightarrow X$ is non-singular: inverse images of sets of zero measure are of zero measure. These measures are supposed to 
be invariant by the flows $\widehat{\phi}_{t}$ and $\phi_{t}$; the space $\widehat{X}$ admits a countable cover by open sets of finite $\widehat{\mu}$-measure, whereas $\mu$ is a probability measure ergodic with respect to the flow $\phi_{t}$. The measure $\widehat{\mu}$ satisfies the following conditions: there is a neighborhood of the identity in $G$ and a constant $C$ such that $g_{*} \widehat{\mu} \leq C \widehat{\mu}$ for $g$ in the neighborhood. We also suppose:

Given a periodic vector $v$ in $X$, let $V$ be a neighborhood of $\pi^{-1} v$ coming from the local product structure. Consider the set $A$ of points $\widehat{x}$ in $V$, for which $[v]^{-1} \widehat{\phi}_{\ell(v)}(\widehat{x})$ is again in $V$ and $\delta_{1}$-close to $\widehat{x}$, and the transformation $T$ on $A$ which associate to the point $\widehat{x}$ in $A$ the point $\left\langle[v]^{-1} \widehat{\phi}_{\ell(v)}(\widehat{x}), \widehat{x}\right\rangle$. Then we suppose that there is a constant $C$ depending only on $v$, such that $\widehat{\mu}\left(T^{-1} B \cap A\right) \leq C \widehat{\mu}(B)$ for all Borel set $B$ in $\widehat{X}$.

This condition may seem awkward; in practice, it follows easily from the absolute continuity of the measure with respect to the holonomy of the foliations. However, since no regularity on the foliations is assumed, one has to give a more technical statement.

Finally, recall that the partition of $\widehat{X}$ by the stable sets $\widehat{W}^{s s}$ is said to be ergodic if any union of elements of the partition that forms a Borel set is of measure 0 or with complement of measure 0 . In the following, this partition will be called abusively a foliation, and the sets $\widehat{W}^{s s}$ will be called the leaves of the foliation.

We can now state the main result:

Theorem 1. - Let $G$ be a Polish group acting continuously on a Polish space $\widehat{X}$ and $\widehat{\phi}_{t}$ a continuous flow on $\widehat{X}$ commuting with the $G$ action. The system satisfies the previous hypothesis: three uniformity conditions, local contraction of $\widehat{W}^{\text {su }}$ uniformly in fibers, and local product structure. Moreover $X$ and $\widehat{X}$ are equipped with measures $\mu$ and $\widehat{\mu}$ as defined above.

Suppose that $G$ is a nilpotent group admitting a central series composed of closed subgroups $\{1\}=G_{0} \subset G_{1} \subset \cdots \subset G_{n}=G$ so that, for all $i \geq 1$, the subgroup of $G_{i} / G_{i-1}$ generated by the Frobenius elements in $G_{i}$ of periodic orbits of $\phi_{t}$ is dense in $G_{i} / G_{i-1}$ :

$$
\overline{\left\langle\left\{[\tau] ; \tau \text { periodic orbit of } \phi_{t} \text { with }[\tau] \in G_{i}\right\}\right\rangle}=G_{i} / G_{i-1},
$$

then the weak stable foliation $\widehat{W}^{\text {ws }}$ is ergodic with respect to $\widehat{\mu}$.

If moreover the following is satisfied:

$$
\{0\} \times G_{i} / G_{i-1} \subset \overline{\left\langle\left\{(\ell(\tau),[\tau]) ; \tau \text { periodic orbit of } \phi_{t} \text { with }[\tau] \in G_{i}\right\}\right\rangle},
$$

$$
\overline{\left\langle\left\{\ell(\tau) ; \tau \text { periodic orbit of } \phi_{t}\right\}\right\rangle}=\mathbb{R}
$$

then the strong stable foliation $\widehat{W}^{s s}$ is ergodic with respect to $\widehat{\mu}$.

TOME $131-2003-\mathrm{N}^{\mathrm{O}} 2$ 
REMARKs. - - The periodic orbits of $\phi_{t}$ with Frobenius elements in $G_{i}$ are precisely the orbits whose lifts in $\widehat{X} / G_{i}$ are again periodic.

- The conditions for the ergodicity of the strong stable foliation can be rewritten as $\overline{\left\langle\left\{[\tau]^{\prime} ;[\tau]^{\prime} \in G_{i}^{\prime}\right\}\right\rangle}=G_{i}^{\prime} / G_{i-1}^{\prime}$ for all $i \geq 1$, with the convention $G^{\prime}=\mathbb{R} \times G, G_{i}^{\prime}=\{0\} \times G_{i}^{\prime}$ if $i<n$, and $[\tau]^{\prime}=(\ell(\tau),[\tau])$.

- There are systems satisfying the equalities $\left\{[\tau] ;[\tau] \in G_{i}\right\}=G_{i} / G_{i-1}$ and $\overline{\left\langle\left\{\ell(\tau) ;[\tau] \in G_{i}\right\}\right\rangle}=\mathbb{R}$, for all $i \geq 1$, and whose strong stable foliation is not ergodic. An example is provided in the section dealing with subshifts of finite type. In that example, the flow $\widehat{\phi}_{t}$ has no periodic orbits on $\widehat{X}$.

- If the subgroup generated by the lengths of the periodic orbits of $\widehat{\phi}_{t}$ on $\widehat{X}$ is dense in $\mathbb{R}$, then the conditions $\overline{\left\langle\left\{[\tau] ;[\tau] \in G_{i}\right\}\right\rangle}=G_{i} / G_{i-1}$, for all $i$, are easily seen to imply the ergodicity of both foliations on $\widehat{X}$.

A similar result can be proven for transformations instead of flows; here are the modifications to introduce in that case: the group $\mathbb{R}$ should be replaced by $\mathbb{Z}$ whenever it occurs; in the definition of the local product structure, the constant $\delta_{2}$ is removed and $t(x, y)$ is equal to zero. The statement of the theorem now translates verbatim, and there is essentially no change in the proof.

\section{Proof of the ergodicity}

This section is devoted to the proof of the main theorem. The central series of $G$ gives a sequence of systems:

$$
\widehat{X} \rightarrow \widehat{X} / G_{1} \rightarrow \cdots \rightarrow \widehat{X} / G_{i-1} \rightarrow \widehat{X} / G_{i} \rightarrow \cdots \rightarrow \widehat{X} / G_{n-1} \rightarrow \widehat{X} / G=X .
$$

Ergodicity of the strong stable foliation is proven by a recurrence on this series. Assuming that the strong stable foliation of the quotient flow on $\widehat{X} / G_{i}$ is ergodic, one has to show that it is again ergodic on $\widehat{X} / G_{i-1}$. This amounts to showing that Borel functions $F: \widehat{X} / G_{i-1} \rightarrow \mathbb{R}$ invariant by the strong stable foliation of the quotient flow on $\widehat{X} / G_{i-1}$ are invariant by the action of $G_{i} / G_{i-1}$; indeed this implies that $F$ factorizes through an invariant function defined on $\widehat{X} / G_{i}$, and is thus constant by the recurrence hypothesis.

The following set is easily seen to be a closed subgroup of $\mathbb{R} \times G / G_{i-1}$ :

$$
\left\{(t, g) ; \text { for all } \widehat{W}^{\text {ss }} \text { invariant } F \text { in } L^{\infty}\left(\widehat{X} / G_{i-1}\right), F\left(\widehat{\phi}_{-t} g x\right)=F(x)\right\}
$$

The invariant functions will factorize through $\widehat{X} / G_{i}$ if $\{0\} \times G_{i} / G_{i-1}$ is included in this set. In order to obtain this inclusion, it will be shown that the lengths and the Frobenius elements $(\ell(\tau),[\tau])$ of the periodic orbits $\tau$ of $\phi_{t}$ are included in this set, if $[\tau]$ belongs to $G_{i}$.

LEMMA 1. - The closed group

$$
\left\{(t, g) \in \mathbb{R} \times G / G_{i-1} ; \forall F, \widehat{W}^{s s} \text { invariant on } \widehat{X} / G_{i-1}, F\left(\widehat{\phi}_{-t} g x\right)=F(x)\right\}
$$


contains the elements of the form $(\ell(\tau),[\tau])$, where $\tau$ is any periodic orbit of $\phi_{t}$ on $X$, whose Frobenius element belongs to the center of $G / G_{i-1}$.

This will imply the theorem.

Indeed, $G_{i} / G_{i-1}$ is included in the center of $G / G_{i-1}$, since $G$ is nilpotent. The hypothesis of Theorem 1 imply that the closed group mentionned in Lemma 1 contains $\{0\} \times G_{i} / G_{i-1}$. Hence, functions on $\widehat{X}$ invariant by $\widehat{W}^{s s}$, are lifts of functions on $X$ invariant by $W^{s s}$. Moreover, if the condition $\overline{\langle\{\ell(\tau)\}\rangle}=\mathbb{R}$ is satisfied, then functions on $X$, which are $W^{s s}$-invariant, are invariant by the flow: this is the case $G_{i-1}=G$ in Lemma 1. So, these functions are constant, by ergodicity of $\phi_{t}$.

The case of the foliation $\widehat{W}^{w s}$ is similar; the $\mathbb{R}$-component of the group mentioned in Lemma 1 can be dropped, since functions invariant by the weak stable foliation are invariant by the flow $\widehat{\phi}_{t}$ itself.

In order to simplify the notations, the space $\widehat{X} / G_{i-1}$ will be denoted by $\widetilde{X}$ and the quotient flow by $\widetilde{\phi}_{t}$. The central series then gives the sequence:

$$
\widetilde{X} \longrightarrow \tilde{X} /\left(G_{i} / G_{i-1}\right) \longrightarrow \widetilde{X} /\left(G / G_{i-1}\right)=X .
$$

3.1. Deformation along periodic orbits. - Let $v \in X$ a periodic point of $\phi_{t}$, whose Frobenius element $[v]$ belongs to $G_{i}$. Since the group $G_{i} / G_{i-1}$ is abelian, the image of $[v]$ in this quotient is uniquely defined. Given some point $\widetilde{x} \in \widetilde{X}$, we explain how to build a point $\widetilde{y}$ belonging to $\widetilde{W^{s s}}(\widetilde{x})$ so that the point $\widetilde{\phi}_{\ell(v)}[v]^{-1}(\widetilde{y})$ is arbitrarily close to $\widetilde{x}$. This will be enough to insure the ergodicity of $\widetilde{W}^{s s}$.

Let $\varepsilon>0$. By conservativity and ergodicity of $\phi_{t}$, almost every point $\widetilde{x} \in \widetilde{X}$ has a projection $\pi(\widetilde{x})$ in $X$ which has a positively transitive orbit. In particular, the orbit of $\pi(\widetilde{x})$ comes arbitrarily close to $v$. This means that the orbit of $\widetilde{x}$ comes close to some preimage $\widetilde{v} \in \widetilde{X}$ of $v$. By continuity of the flow, it stays close to the orbit of $\widetilde{v}$ during a time equal to, say, twice the period of $v$. So, for almost all $\widetilde{x} \in \widetilde{X}$, and any $\varepsilon^{\prime}>0$, there exists an $n \in \mathbb{N}$ such that $d\left(\widetilde{\phi}_{n \ell(v)+t}(\widetilde{x}), \widetilde{\phi}_{t}(\widetilde{v})\right)<\varepsilon^{\prime}$, for all positive $t$ less than or equal to $\ell(v)$. The set of such elements $\widetilde{x}$ is denoted by $\widetilde{D}_{n}$; These Borel sets cover almost all of $\widetilde{X}$. For the points in $\widetilde{D}_{n}$, we have:

$$
d\left(\widetilde{\phi}_{n \ell(v)}(\widetilde{x}), \widetilde{v}\right)<\varepsilon^{\prime}, \quad d\left(\widetilde{\phi}_{n \ell(v)+\ell(v)}(\widetilde{x}), \widetilde{\phi}_{\ell(v)}(\widetilde{v})\right)<\varepsilon^{\prime} .
$$

By definition of the Frobenius element, $\widetilde{\phi}_{\ell(v)}(\widetilde{v})=[v](\widetilde{v})$. Moreover, $[v]$ acts uniformly on the fiber of $\widetilde{v}$; given some $\delta_{1}>0$, one can choose $\varepsilon^{\prime}$ such that

$$
d\left(\widetilde{\phi}_{n \ell(v)}(\widetilde{x}),[v]^{-1} \widetilde{\phi}_{\ell(v)} \widetilde{\phi}_{n \ell(v)}(\widetilde{x})\right) \leq \varepsilon .
$$

The local product structure can now be used to get a real number $t$ close to 0 , an element $g$ of $G$ close to identity, and a point $\widetilde{z}$ close to $\widetilde{v}$ which lies both in

TOME $131-2003-\mathrm{N}^{\mathrm{O}} 2$ 
$\widetilde{W}_{\varepsilon}^{s u}\left(\widetilde{\phi}_{n \ell(v)}(\widetilde{x})\right)$ and in $\widetilde{W}_{\varepsilon}^{s s}\left([v]^{-1} \widetilde{\phi}_{\ell(v)} \widetilde{\phi}_{n \ell(v)} g \widetilde{\phi}_{t}(\widetilde{x})\right)$. One then take

$$
\widetilde{y}=[v] \widetilde{\phi}_{-\ell(v)} g^{-1} \widetilde{\phi}_{-n \ell(v)-t}(\widetilde{z}) .
$$

The point $\widetilde{y}$ is in $\widetilde{W}^{s s}(\widetilde{x})$ and satisfies:

$$
[v]^{-1} \widetilde{\phi}_{\ell(v)} g \widetilde{\phi}_{t}(\widetilde{y}) \in \widetilde{W}^{s u}(\widetilde{x}), \quad d\left(g \widetilde{\phi}_{t}[v]^{-1} \phi_{\ell(v)}(\widetilde{y}), \widetilde{x}\right) \leq \varepsilon .
$$

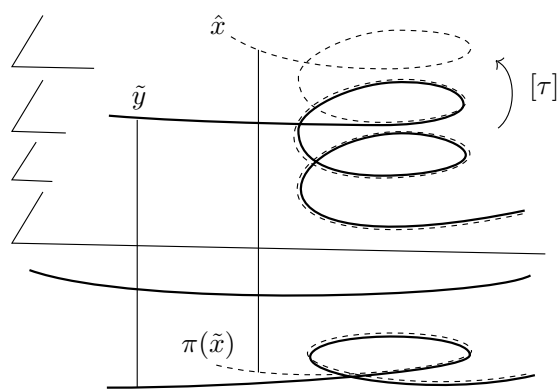

FiguRE 1. Deformation of the orbit

3.2. Ergodicity. - We now suppose that the element $(\ell(v),[v])$ does not belong to the subgroup

$$
\left\{(t, g) \in \mathbb{R} \times G / G_{i-1} ; \forall F \in L^{\infty}(\widetilde{X}), F \text { invariant, } F\left(\widetilde{\phi}_{-t} g x\right)=F(x)\right\}
$$

and try to get a contradiction.

We first note that we may restrict our attention to functions $F$ satisfying the following continuity assumption:

$$
\begin{aligned}
& \forall \eta>0, \exists r>0, \forall(t, g) \in \mathbb{R} \times G \text { with } d((t, g),(0, \text { id }))<r, \\
& \forall \widetilde{x} \in \widetilde{X},\left|F\left(g \widetilde{\phi}_{t}(\widetilde{x})\right)-F(\widetilde{x})\right|<\frac{1}{2} \eta .
\end{aligned}
$$

Indeed, any bounded measurable function $F$ may be approximated by such a function, using the formula:

$$
F * \theta(\widetilde{x})=\int_{\mathbb{R} \times G} F\left(h \phi_{s} \widetilde{x}\right) \theta(s, h) \mathrm{d} s \mathrm{~d} h
$$

where $\theta$ is some continuous positive function on $\mathbb{R} \times G$ with integral one and support in a small neighborhood of $(0, \mathrm{id})$. It is elementary to show that the function $F * \theta$ satisfies the continuity assumption above; one also checks that the invariance of $F$ with respect to the foliation implies the same invariance for $F * \theta$. Now, if the functions of the form $F * \theta$ are invariant by the flow and the group action, the same is true for $F$ :

BULletin DE LA SOCIÉtÉ MATHÉMATIQUE DE FRANCE 
LEMmA 2. - There exists functions $\theta_{n}$ such that the sequence $F * \theta_{n}$ converges almost everywhere to $F$.

Proof. - First note that since $\widetilde{X}$ can be covered by countably many open sets of finite measure, $\widetilde{X}$ may be written as a union of a set with zero measure and of countably many compact sets, each of them admitting a neighborhood of finite measure. Let $K$ be one of these compact sets. Then, we have

$$
\int_{K}|F * \theta-F| \longrightarrow 0
$$

as the support of $\theta$ shrinks to $(0, \mathrm{id})$. This is seen by approximating $F$ by a continuous function on a neighborhood of $K$ and using the inequality $g_{*} \widetilde{\mu}<C \widetilde{\mu}$ for $g$ close to the identity. One then chooses a sequence of functions $\theta$ with support decreasing to zero, and extracts subsequences.

So, in order to get a contradiction, we suppose that there exists some function $F \in L^{\infty}(\widetilde{X})$ satisfying the above continuity assumption, invariant by the stable foliation, and that there also exists a Borel set $B$ of positive measure, and some constant $\eta>0$ such that $F\left(\widetilde{\phi}_{-\ell(v)}[v](\widetilde{x})\right)>F(\widetilde{x})+\eta$, for all $\widetilde{x} \in B$. This implies that:

$$
F\left(\widetilde{\phi}_{-\ell(v)}[v](\widetilde{x})\right)>F(\widetilde{y})+\eta, \quad \text { for all } \widetilde{x} \in B, \text { for all } \widetilde{y} \in \widetilde{W}^{s s}(\widetilde{x}) .
$$

Considering $F \circ \phi_{-\ell(v)}[v]$ instead of $F, \widetilde{\phi}_{\ell(v)}[v]^{-1}(B)$ instead of $B$, one gets $F(\widetilde{x})>F\left(\widetilde{\phi}_{\ell(v)}[v]^{-1} \widetilde{y}\right)+\eta$, for all $\widetilde{x} \in B$, for all $\widetilde{y} \in \widetilde{W}^{s s}(\widetilde{x})$. Since $\widetilde{X}$ can be covered by a countable cover of open sets of finite measure, the measure $\widetilde{\mu}$ is regular and $B$ can be taken compact, with a $\delta$-neighborhood of finite measure.

Restricting $B$ slightly and applying Luzin theorem, one can suppose that $F$ is uniformly continuous on $B$ : there is an $\varepsilon>0$ such that if $\widetilde{x}, \widetilde{x}^{\prime} \in B, d\left(\widetilde{x}, \widetilde{x}^{\prime}\right)<\varepsilon$, then $\left|F(\widetilde{x})-F\left(\widetilde{x}^{\prime}\right)\right|<\frac{1}{2} \eta$.

So if there exists $\widetilde{x} \in B, \widetilde{y} \in \widetilde{W}^{s s}(\widetilde{x})$ such that $[v]^{-1} \widetilde{\phi}_{\ell(v)} g \widetilde{\phi}_{t}(\widetilde{y})$ is in $B$ and at distance less than $\varepsilon$ from $\widetilde{x}$, one can bound $\left|F(\widetilde{x})-F\left([v]^{-1} \widetilde{\phi}_{\ell(v)}(\widetilde{y})\right)\right|$ by

$$
\left|F(\widetilde{x})-F\left(g \widetilde{\phi}_{t}[v]^{-1} \widetilde{\phi}_{\ell(v)}(\widetilde{y})\right)\right|+\left|F\left(g \widetilde{\phi}_{t}[v]^{-1} \widetilde{\phi}_{\ell(v)}(\widetilde{y})\right)-F\left([v]^{-1} \widetilde{\phi}_{\ell(v)}(\widetilde{y})\right)\right| .
$$

The first term is bounded by $\frac{1}{2} \eta$ by uniform continuity, and the second term is bounded by $\frac{1}{2} \eta$ by the continuity assumption on $F$. This gives a contradiction.

The point $\widetilde{y}$ is built by deformation along the periodic orbit, using the $\varepsilon$ giving the contradiction in the previous argument. It remains to show that $[v]^{-1} \widetilde{\phi}_{\ell(v)} g \widetilde{\phi}_{t}(\widetilde{y})$ can be taken in $B$. This is the goal of the next section.

3.3. Return times. - Recall that the sets $\widetilde{D}_{k}, k \in \mathbb{N}$, are the sets of points $\widetilde{x} \in \widetilde{X}$ which enter a sufficiently small neighborhood of the fiber of $\widetilde{v}$ at time $k \ell(v)$. Note also that there is an $\varepsilon^{\prime}>0$ such that each of the sets $\pi^{-1}\left(\phi_{t}^{-1}\left(B\left(v, \varepsilon^{\prime}\right)\right)\right), t>0$, is contained in some $\widetilde{D}_{k}$. The sets $\widetilde{D}_{k}$ cover almost 
all of $\widetilde{X}$. The point $[v]^{-1} \widetilde{\phi}_{\ell(v)} g \widetilde{\phi}_{t}(\widetilde{y})$ associated to $\widetilde{x}$ via deformation is denoted by $T_{k}(\widetilde{x})$. It is equal to $\widetilde{\phi}_{-k \ell(v)}(\widetilde{z})$, where $\widetilde{z}$ is the point near $\widetilde{v}$ obtained by using the local product structure. In order to complete the proof of the theorem, it is sufficient to find an $\widetilde{x} \in B$ and an integer $k$ such that $\widetilde{x}$ is in $\widetilde{D}_{k}$ and $T_{k}(\widetilde{x})$ is in $B$. This is the goal of the next two lemmas.

Lemma 3. - $\widetilde{\mu}\left(\left\{\widetilde{x} \in B ; \widetilde{x} \in \widetilde{D_{k}}, T_{k}(\widetilde{x}) \notin B\right\}\right) \rightarrow 0$ as $k \rightarrow \infty$.

Proof. - Recall that $V_{\delta} B$ is the $\delta$-neighborhood of $B$. Since $B$ is a closed set, the quantities $\widetilde{\mu}\left(V_{\delta} B \backslash B\right)$ tend to zero as $\delta$ tends to zero. So, given some $\eta>0$, there exists a $\delta_{0}>0$ such that $\widetilde{\mu}\left(V_{\delta_{0}} B \backslash B\right)<\eta / C$. Moreover, $T_{k}(\widetilde{x})$ is on $W^{s u}(\widetilde{x})$ and the images of these two points get $\varepsilon$-close after time $k$. Since the unstable foliation is locally contracting uniformly in fibers, there is a $k_{0}$ such that for all $k \geq k_{0}$, and all $\widetilde{x} \in \widetilde{D}_{k}, d\left(\widetilde{x}, T_{k}(\widetilde{x})\right)<\delta_{0}$. The transformation $T_{k}$ is obtained by composition of $\widetilde{\phi}_{k l(v)}$ with the deformation process in the local product neighborhood of some preimage of $v$. The measure $\widetilde{\mu}$ is invariant by the flow and satisfies a uniform majoration under the deformation; this gives:

$$
\widetilde{\mu}\left(T_{k}^{-1}\left(V_{\delta_{0}} B \backslash B\right) \cap \widetilde{D}_{k}\right) \leq C \widetilde{\mu}\left(V_{\delta_{0}} B \backslash B\right) \leq \eta .
$$

Finally if $k \geq k_{0}$ the set $\left\{\widetilde{x} \in B ; \widetilde{x} \in \widetilde{D}_{k}, T_{k}(\widetilde{x}) \notin B\right\}$ is contained in the set $T_{k}^{-1}\left(V_{\delta_{0}} B \backslash B\right) \cap \widetilde{D}_{k}$.

LemMA 4. - $\widetilde{\mu}\left(\left\{\widetilde{x} \in B ; \widetilde{x} \in \widetilde{D_{k}}\right\}\right) \nrightarrow 0$ as $k \rightarrow \infty$.

Proof. - If the quantities $\widetilde{\mu}\left(B \cap \pi^{-1} \phi_{-t} B\left(v, \varepsilon^{\prime}\right)\right)$ tend to zero as $t$ tends to infinity, the same is true of the integral:

$$
\frac{1}{t} \int_{0}^{t} \int_{B} \mathbf{1}_{B(v, \varepsilon)} \circ \phi_{s} \mathrm{~d}\left(\pi_{*} \widetilde{\mu}\right) \mathrm{d} s .
$$

But this integral converges to $\mu\left(B\left(v, \varepsilon^{\prime}\right)\right) \widetilde{\mu}(B)$ : this is a direct application of the Fubini theorem, the Birkhoff ergodic theorem and the Lebesgue dominated convergence theorem. This proves the lemma.

\section{Hyperbolic systems}

In this section, the main result is applied to hyperbolic flows and diffeomorphisms.

Let $\phi_{t}$ be a $C^{1}$ flow on a $C^{\infty}$ riemannian manifold $M$ and $\Lambda$ a compact set invariant by $\phi_{t}$.

Definition 1. - The flow is said to be hyperbolic on $\Lambda$ if $\Lambda$ contains no fixed points of the flow, periodic points are dense in $\Lambda$, there exists an orbit dense in $\Lambda$, and there is an open set $U$ containing $\Lambda$ such that $\bigcap \phi_{t} U=\Lambda$, the 
intersection being on all $t \in \mathbb{R}$ and, moreover, if there exist positive constants $C, \lambda$, such that for all $x \in \Lambda$ there is a decomposition of the tangent bundle $T_{x} M=E_{x}^{0} \oplus E_{x}^{s s} \oplus E_{x}^{s u}$ satisfying:

- $E^{0}$ is one-dimensional tangent to the flow;

- $D \phi_{t} E_{x}^{s s}=E_{x}^{s s}, D \phi_{t} E_{x}^{s u}=E_{x}^{s u}$;

- $\left\|D \phi_{t \mid E_{x}^{s s}}\right\| \leq C \mathrm{e}^{-\lambda t}$ and $\left\|D \phi_{-t \mid E_{x}^{s u}}\right\| \leq C \mathrm{e}^{-\lambda t}$ for all $t>0$, .

There is a similar definition for diffeomorphisms; the $E^{0}$ part has to be removed in the previous statement; see, e.g. [25].

Transitive Anosov flows correspond to flows hyperbolic on the whole manifold. Axiom A flows are a specific class of flows which are hyperbolic on the components of the non-wandering set.

Hyperbolic flows admit many invariant ergodic probability measures. Given some Hölder continuous function $f$ on $\Lambda$, there is a unique measure maximizing the quantity $h_{\mu}+\int f \mathrm{~d} \mu$ among the set of invariant Borel probability measures $\mu$ supported on $\Lambda$, where $h_{\mu}$ is the entropy of $\mu$ with respect to $\phi_{1}$ [7]. This measure is called the Gibbs measure associated to the potential $f$. We will check below that it satisfies the conditions of Theorem 1 .

Let $\phi_{t}$ a hyperbolic flow on a compact set $\Lambda \subset M, \widehat{M}$ a $G$-fiber bundle over $M$ and $\widehat{\phi}_{t}$ an extension of $\phi_{t}$ on $\widehat{M}$ commuting with the $G$-action. In local trivializations $U \times G, \widehat{\phi}_{t}$ takes the form $(x, g) \rightarrow\left(\phi_{t}(x), A_{t}(x) g\right)$, where $A_{t}$ is an map from $U$ to $G$. We say that $\widehat{\phi}_{t}$ is a Hölder extension of $\phi_{t}$ if there is a covering of $\Lambda$ with trivializations charts such that the maps $A_{t}(x)$ are Hölder continuous in $x$ for small $t$. Note that when $G$ is discrete, there exists a unique extension, which corresponds to $A_{t} \equiv 0$. In the case of diffeomorphisms, an extension is said to be Hölder if it is of the form $(x, g) \mapsto(\phi(x), f(x) g)$ in local charts $U \times G$, with $f: U \rightarrow G$ Hölder.

THEOREM 2. - Let $\phi_{t}$ (resp. $\left.\phi\right)$ an hyperbolic flow (resp. diffeomorphism) on some compact subset $\Lambda$ of a manifold $M, G$ a locally compact second countable group carrying a biinvariant distance, $\widehat{M}$ a Hölder principal G-fiber bundle over $M, \widehat{\phi}_{t}$ (resp. $\left.\widehat{\phi}\right)$ a Hölder extension of $\phi_{t}$ (resp. $\left.\phi\right)$ to $\widehat{M}$ commuting with $G, \mu$ a Gibbs measure on $\Lambda$. The manifold $\widehat{M}$ is endowed with a measure $\widehat{\mu}$ which coincides with $\mu \times$ Haar on local trivializations of the bundle. Suppose that $G$ is a nilpotent group admitting a central series composed of closed subgroups $\{1\}=G_{0} \subset G_{1} \subset \cdots \subset G_{n}=G$ such that:

$$
\overline{\left\langle\left\{[\tau] ; \tau \text { periodic orbit on } \Lambda \text { with }[\tau] \in G_{i}\right\}\right\rangle}=G_{i} / G_{i-1} \text {, }
$$

then the weak stable foliation $\widehat{W}^{w s}$ is ergodic with respect to $\widehat{\mu}$. If moreover

$$
\begin{aligned}
\{0\} \times G_{i} / G_{i-1} \subset \overline{\left\langle\left\{(\ell(\tau),[\tau]) ; \tau \text { periodic orbit on } \Lambda \text { with }[\tau] \in G_{i}\right\}\right\rangle}, \\
\qquad \overline{\langle\{\ell(\tau) ; \tau \text { periodic orbit on } \Lambda\}\rangle}=\mathbb{R} \quad(\text { resp. } \mathbb{Z}),
\end{aligned}
$$

TOME $131-2003-\mathrm{N}^{\mathrm{O}} 2$ 
then the strong stable foliation $\widehat{W}^{s s}$ is ergodic with respect to $\widehat{\mu}$.

REMARK. - There are similar statements for $\widehat{W}^{s u}, \widehat{W}^{w s}, \widehat{W}^{w u}$, which follow from Theorem 1 . Note that the condition $\overline{\langle\{\ell(\tau)\}\rangle}=\mathbb{R}$ (resp. $\mathbb{Z}$ ) is equivalent to the flow (resp. the diffeomorphism) being topologically mixing on $\Lambda$. It is always satisfied for hyperbolic diffeomorphisms if $\Lambda$ is connected; on the other hand, it fails for flows which are constant suspension of diffeomorphisms.

In order to prove this result, we have to show that the hypothesis of Theorem 1 are satisfied for these systems; this is checked in three steps.

The case $G=\{0\}$. - In the context of Axiom A flows, local product structures were introduced by S. Smale [26]. The next result is taken from R. Bowen [5].

Proposition 1. - Let $\phi_{t}$ be a hyperbolic flow on some compact $\Lambda$; there are $\delta_{1}, \delta_{2}$ for which the following is true: if $x, y \in \Lambda$ and $d(x, y) \leq \delta_{1}$, then there is a unique $t=t(x, y)$ with $|t| \leq \delta_{2}$ so that

$$
W_{\delta_{2}}^{s s}\left(\phi_{t}(x)\right) \cap W_{\delta_{2}}^{s u}(y)=\langle x, y\rangle .
$$

This set consists of a single point, which is denoted by $\langle x, y\rangle$. The maps $t$ and $\langle$,$\rangle are continuous on the set \left\{(x, y) ; d(x, y) \leq \delta_{1}\right\} \subset X \times X$.

A rectangle is a compact set, equal to the closure of its interior, such that the local product of two points in the rectangle is again a rectangle. Each point of $\Lambda$ has a basis of neighborhoods composed of rectangles. This gives the local product structure we need.

In the case of diffeomorphisms, there is a similar result; in fact, spaces admitting maps with local product structures are called Smale spaces; their study is developed in [24], where it is shown that they are essentially equivalent to subshifts of finite type.

Now a Gibbs measure $\mu$ can be written as a product of a family $\mu^{s u}$ of transverse measures to the strong unstable foliation by a family of measures $\mu^{s}$ supported by the strong unstable leaves; moreover the modulus of absolute continuity of these families with respect to the holonomy of the foliations is explicitly known:

$$
\frac{\mathrm{d} \zeta_{*} \mu_{A}^{s u}}{\mathrm{~d} \mu_{B}^{s u}}(y)=\mathrm{e}^{\left[\zeta^{-1} y, y\right]}
$$

where $A, B$ are any pairs of transversals to $W^{s u}, \zeta$ any one-to-one mesurable map such that $\zeta(x) \in W^{s u}(x)$ for almost all $x \in A$, and $[x, y]$ is defined by

$$
[x, y]=\lim _{t \rightarrow-\infty} \int_{0}^{t} f\left(\phi_{s} x\right) \mathrm{d} s-\int_{0}^{t} f\left(\phi_{s} y\right) \mathrm{d} s, \text { for all } x \in W^{s u}(y),
$$

BULLETIN DE LA SOCiÉtÉ MATHÉMATIQUE DE FRANCE 
where $f$ is a Hölder function on $M$ called the potential of the measure $\mu$. Note that this limit is converging because $f$ is Hölder and $\phi_{-t}$ is exponentially contracting. The bracket $[x, y]$ is thus bounded by a constant depending on the Hölder constants of $f$, the contraction exponent of the flow, and the diameter of $\Lambda$, but not on $x, y$. There are similar estimates for the modulus of absolute continuity of $\mu^{s}$ with respect to the action of $\phi_{\ell}$ and the holonomy of the weak stable foliation. We refer to [3], Lemma 1, for explicit formulas. The point is that these modulus are uniformly bounded on $\Lambda$.

The estimate needed on the transformation $T$, which sends the point $x$ to $\left\langle\phi_{\ell}(x), x\right\rangle$, follows from these properties. Given some point $x$ in a rectangle, $T$ sends the local weak stable leaf $W_{\varepsilon}^{w s}(x)$ to $W^{w s}\left(\phi_{\ell}(x)\right)$; in restriction to this leaf, it coincides with the holonomy map $\zeta$ between the two transversals $W_{\varepsilon}^{w s}(x)$ and $W^{w s}\left(\phi_{\ell}(x)\right)$. Hence, $T$ has a bounded modulus of absolute continuity on $W_{\varepsilon}^{w s}(x)$ with respect to $\mu^{s u}$, and this modulus does not depend on $x$. On the other end, the fibers $W^{s u}(x)$ are send to themselves by $T$; the image of a point $y$ in $W^{s u}(x)$ is obtained first by applying $\phi_{\ell}$ from $W^{s u}(x)$ to $W^{s u}\left(\phi_{\ell}(x)\right)$, then by following the weak stable leaves to return to $W^{s u}(x)$. The modulus of absolute continuity is again bounded independently of $x$, so we are done.

The case $G$ discrete. - Principal bundles with discrete structure group correspond to regular covers. The data on $X$ lift to $\widehat{X}$ : given some point $v$, one considers a rectangle contained in some neighborhood $U$ trivializing the covering; the inverse image of this neighborhood is of the form $U \times G$ and the local product structure is defined by $\langle(x, g),(y, g)\rangle=(\langle x, y\rangle, g)$. The properties of the measure $\widetilde{\mu}$ then follows from the one of $\mu$, since $\widetilde{\mu}$ is equal to $\mu$ on each level of the cover.

The general case. - In order to pull back the local product structure from $\Lambda$ to $\pi^{-1} \Lambda$, we show how to find for any two close points $x, y$ on $\Lambda$ such that $x \in W^{s s}(y)$, an element $g(x, y)$ with $(x, g(x, y)) \in \widehat{W}^{s s}((y, \mathrm{id}))$.

The compact $\Lambda$ is first covered with a finite number of flow boxes trivializing the fiber bundle. There is an $\epsilon$ such that for any point $x$ in $\Lambda, \phi_{[-\varepsilon, \varepsilon]}(B(x, \varepsilon))$ is contained in one of the previous flow boxes. The orbit of some point $\widehat{x}$ in the fiber of $x$ can be described by using a succession of such sets: the point first travels during time $\varepsilon$ through the first trivialization; one then changes from trivialization to travel through another during time $\varepsilon$, and so on. The expression of $\widehat{\phi}_{n \epsilon}(x, g)$ takes the form $\left(\phi_{n \varepsilon}(x), \prod_{k=0}^{n} g_{k}\left(\phi_{k \varepsilon}(x)\right) A_{\varepsilon}^{k}\left(\phi_{k \varepsilon}(x) g\right)\right.$, where the $g_{k}$ correspond to the change of trivialization at time $k \varepsilon$ and the $A^{k}$ refer to the expression of the flow in that trivialization. Note that the $g_{k}{ }^{\prime} s$ and the $A^{k \prime} s$ are commuting with each others. 
If $y$ belongs to $W_{\varepsilon}^{s s}(x)$, the displacement in the fibers between the orbits of $\widehat{\phi}_{n \varepsilon}(x, \mathrm{id})$ and $\widehat{\phi}_{n \varepsilon}(y, \mathrm{id})$ is equal to

$$
\prod_{k=0}^{n} g_{k}\left(\phi_{k \varepsilon}(y)\right) A_{\varepsilon}^{k}\left(\phi_{k \varepsilon}(y)\right)\left(\prod_{k=0}^{n} g_{k}\left(\phi_{k \varepsilon}(x)\right) A_{\varepsilon}^{k}\left(\phi_{k \varepsilon}(x)\right)\right)^{-1} .
$$

The biinvariance of the distance on $G$ implies the inequality

$$
d\left(g_{1} g_{2}, g_{3} g_{4}\right) \leq d\left(g_{1}, g_{3}\right)+d\left(g_{2}, g_{4}\right) .
$$

Since there are a finite number of flow boxes, the $g_{k}{ }^{\prime} s$ and the $A^{k \prime} s$ are Hölder continuous, and the flow is exponentially contracting, the series $\sum d\left(g_{k}\left(\phi_{k \varepsilon}(x)\right) A_{\varepsilon}^{k}\left(\phi_{k \varepsilon}(x)\right), g_{k}\left(\phi_{k \varepsilon}(y)\right) A_{\varepsilon}^{k}\left(\phi_{k \varepsilon}(y)\right)\right)$ is uniformly convergent, and bounded by a constant times $d(x, y)$. This shows that the translation in the fiber converges to a continuous function $g(x, y)$. This function tends to zero as $d(x, y)$ tends to zero. There is a similar construction for the strong unstable foliation which gives a function $g^{\prime}(x, y)$ so that $\left(x, g^{\prime}(x, y)\right) \in \widehat{W}^{s u}((y, \mathrm{id}))$.

The local product structure can now be defined by the following formula:

$$
\left\langle\left(x, g_{1}\right),\left(y, g_{2}\right)\right\rangle=\left(\langle x, y\rangle, g(\langle x, y\rangle, y)^{\prime} g_{2}\right) \text {. }
$$

This point belongs to $\widehat{W}^{s u}\left(\left(y, g_{2}\right)\right)$; it also belongs to $\widehat{W}^{s s}\left(\widetilde{g} \widehat{\phi}_{t}\left(x, g_{1}\right)\right)$, where $\widetilde{g}$ is equal to

$$
\widetilde{g}=A_{t}^{-1} g\left(\langle x, y\rangle, \phi_{t}(x)\right) g(\langle x, y\rangle, y)^{\prime} g_{2} g_{1}^{-1},
$$

and $t$ given by the local product structure on $\Lambda$. Note that the element $\widetilde{g}$ is close to the identity, as needed. Moreover, the local product structure acts as a translation in each fiber; since the measure $\widehat{\mu}$ coincides with the Haar measure in the fiber, the estimate needed for the measure $\widehat{\mu}$ follows from the one for $\mu$ and the Fubini theorem.

\section{Subshifts of finite type}

Shifts on subshifts of finite type are essentially the same as hyperbolic diffeomorphisms. On the one hand, hyperbolic diffeomorphisms can be coded by subshifts (see, e.g. [25]), whereas any subshift can be realized as the nonwandering set of some hyperbolic system, the shift being conjugated to the diffeomorphism. They both stand in the category of Smale spaces [24], for which Theorem 1 applies.

A subshift of finite type is given by a finite alphabet $I$ with $n$ elements and a $n \times n$ transition matrix $A$ with coefficients in $\{0,1\}$. The space of sequences of the subshift is defined by

$$
\Sigma_{A}:=\left\{\left\{x_{k}\right\}_{k \in \mathbb{Z}} \in I^{\mathbb{Z}} ; \forall k \in \mathbb{Z}, A_{x_{k} x_{k+1}}=1\right\}
$$

BULletin DE LA SOCIÉtÉ MATHÉMATIQUE DE FRANCE 
and the shift is the homeomorphism on $\Sigma_{A}$ given by $\sigma\left(\left\{x_{i}\right\}\right)_{i}=x_{i+1}$. The distance between two words $x_{i}, y_{i}$ is equal to

$$
d\left(\left\{x_{i}\right\},\left\{y_{i}\right\}\right)=\exp \left(-\min \left\{|i| \text { with } x_{i} \neq y_{i}\right\}\right) .
$$

The local product structure is defined on words with same zero coordinate as

$$
\left\langle\left(\ldots, x_{-1}, x_{0}, x_{1}, \ldots\right),\left(\ldots, y_{-1}, y_{0}, y_{1}, \ldots\right)\right\rangle=\left(\ldots, y_{-2}, y_{-1}, y_{0}, x_{1}, x_{2}, \ldots\right) .
$$

The notion of Gibbs measure is defined in the same way as for hyperbolic diffeomorphisms: given a Hölder function $f$ on $\Sigma_{A}$, there is a unique invariant Borel probability measure maximizing the quantity $h_{\mu}+\int f \mathrm{~d} \mu$, where $h_{\mu}$ denotes the entropy of the measure with respect to the shift. This measure is ergodic, of full support.

Repeating the arguments of the previous section, one gets the following result; V. Kaimanovich and K. Schmidt announced a similar statement under slightly different hypothesis, in [21]:

TheOrem 3. - Let $\left(\Sigma_{A}, \sigma\right)$ a transitive subshift of finite type, $\mu$ a Gibbs measure on $\Sigma_{A}, G$ a locally compact second countable group admitting a biinvariant metric, $f: \Sigma_{A} \rightarrow G$ a Hölder continuous function depending only on positive coordinates, and $T$ the transformation on $\Sigma_{A} \times G$ which sends $(x, g)$ to $(\sigma x, f(x) g)$. Suppose that $G$ is a nilpotent group admitting a central series composed of closed subgroups $\{1\}=G_{0} \subset G_{1} \subset \cdots \subset G_{n}=G$ such that

$$
\overline{\left\langle\left\{[\tau] ; \tau \text { periodic point of } \sigma \text { with }[\tau] \in G_{i}\right\}\right\rangle}=G_{i} / G_{i-1} \text {, }
$$

then the weak stable foliation of $T$ is ergodic with respect to $\mu \times$ Haar. If moreover the following is satisfied

$$
\begin{aligned}
&\{0\} \times G_{i} / G_{i-1} \subset \overline{\left\langle\left\{(\ell(\tau),[\tau]) ; \tau \text { periodic point of } \sigma \text { with }[\tau] \in G_{i}\right\}\right\rangle}, \\
&\langle\{\ell(\tau) ; \tau \text { periodic point of } \sigma\}\rangle=\mathbb{Z},
\end{aligned}
$$

then the strong stable foliation of $T$ is ergodic with respect to $\mu \times$ Haar.

REMARKs. - - The condition $\langle\{\ell(\tau)\}\rangle=\mathbb{Z}$ is equivalent to the shift being topologically mixing.

- The ergodicity of the weak (resp. strong) stable foliation implies the equality $\overline{\langle\{[\tau]\}\rangle}=G$ (resp. $\overline{\langle\{(\ell(\tau),[\tau])\}\rangle}=\mathbb{Z} \times G)$; this is a consequence of the Livsic Theorem [22]; the argument can be found in [10]. In particular, the conditions on the periodic orbits are necessary if $G$ is abelian.

- If the set $\{[\tau]\}$ is invariant by the map $g \mapsto g^{-1}$ and the subshift is a Bernoulli shift, which means that $A_{i, j}=1$, for all $i, j$, then the conditions in term of periodic orbits break down to $\overline{\langle\{[\tau]\}\rangle}=G$ for the weak stable foliation; indeed a product of Frobenius elements of periodic orbits is the Frobenius element of a single orbit, obtained by concatenating the words associated to each orbit, hence commutators of Frobenius elements are again Frobenius elements.

TOME $131-2003-\mathrm{N}^{\mathrm{O}} 2$ 
- Since we have made the assumption that $f$ depends only on positive coordinates, the stable foliation may be described as follows: one writes $f^{m}(x)$ for the product $f\left(\sigma^{m-1} x\right) \cdots f(x)$; then two points $\left(\left\{x_{i}\right\}, u\right),\left(\left\{y_{i}\right\}, v\right)$ are on the same weak (resp. strong) stable leaf if and only if there are two positive integers $m, n$ (resp. $m=n$ ) with $x_{i+m}=y_{i+n}$, for all $i \geq 0$, and $f^{m}(x) u=f^{n}(y) v$.

EXAMPLES. - Let $f:\{0,1\}^{\mathbb{Z}} \rightarrow \mathbb{Z}$ be the map which takes value 1 on the set $\left\{x ; x_{0}=0\right\}$ and -1 on its complementary set. Consider the system $(\sigma, f)$ on $\{0,1\}^{\mathbb{Z}} \times \mathbb{Z}$; its weak stable foliation is ergodic, whereas its strong stable foliation is not. Indeed, $\langle[\tau]\rangle=\mathbb{Z}$ but $\ell(\tau)+[\tau]$ is even, so that $\langle\{(\ell(\tau),[\tau])\}\rangle \neq \mathbb{Z} \times \mathbb{Z}$.

Moreover let $\alpha$ be an irrationnal real number, $r:\{0,1\}^{\mathbb{Z}} \rightarrow \mathbb{R}$ the map which takes value 1 on the set $\left\{x \mid x_{0}=0\right\}$ and $\alpha$ on its complementary set. The space $\{0,1\}^{\mathbb{Z}} \times \mathbb{R}$ can be quotiented by the action $(x, t) \mapsto(\sigma x, t-r(x))$ to obtain a suspension over the subshift $\{0,1\}^{\mathbb{Z}}$. The $\mathbb{R}$-action on the suspension can be conjuguated to a hyperbolic flow on some compact set homeomorphic to the suspension space.

One gets an extension of that system by quotienting $\{0,1\}^{\mathbb{Z}} \times \mathbb{R} \times \mathbb{Z}$ by $(x, t, n) \mapsto(\sigma x, t-r(x), n+f(x))$. Note that ergodicity of the stable foliation of the $\mathbb{R}$-action on the whole space $\{0,1\}^{\mathbb{Z}} \times \mathbb{R} \times \mathbb{Z}$ is the same as ergodicity on the quotient, since two points with the same image in the quotient are on the same stable leaf. We now have:

$$
\overline{\langle\{(\ell(\tau),[\tau])\}\rangle}=\mathbb{Z}(1,1) \oplus \mathbb{Z}(\alpha,-1), \quad \overline{\langle\{\ell(\tau)\}\rangle}=\mathbb{R}, \quad\{[\tau]\}=\mathbb{Z} .
$$

This gives an example of a mixing hyperbolic flow $\phi_{t}$ with extension $\widehat{\phi}_{t}$ satisfying $\{[\tau]\}=G$, for which the strong stable foliation is not ergodic.

\section{Geodesic flow on negatively curved manifolds}

Let $H$ be a connected simply connected Riemannian manifold with sectional curvature bounded between two negative constants. Such a manifold is homeomorphic to a ball and can be compactified by adding a sphere at infinity, which is denoted by $\partial H$; see, e.g. [14]. This boundary may be defined as equivalence classes of asymptotic rays in $H$. Given two different points on $\partial H$, there is exactly one geodesic going from one point to the other. The boundary can be used to get a parametrization of the unitary tangent bundle $S^{1} H$ of $H$, called the Hopf coordinates, from $(\partial H \times \partial H-\Delta) \times \mathbb{R}$ to $S^{1} H$, where $\Delta$ denotes the diagonal $\{(\xi, \eta) ; \xi=\eta\}$. It sends the point $(\xi, \eta, t)$ to the unitary vector tangent to the oriented geodesic from $\xi$ to $\eta$, and whose projection on $H$ is at distance $t$ from an origin on the geodesic; one can take as origin the closest point on the geodesic to a fixed point $x_{0} \in H$, for example.

The geodesic flow on $S^{1} H$ translates vectors along the geodesic they generate. In the Hopf coordinates, this is just the translation on the $\mathbb{R}$-factor. The 
stable foliations of the flow may be described using the Hopf coordinates:

$$
\begin{aligned}
W^{w s}(\xi, \eta, t) & =\left\{\left(\xi^{\prime}, \eta^{\prime}, t^{\prime}\right) ; \eta^{\prime}=\eta\right\}, \\
W^{s s}(\xi, \eta, t) & =\left\{\left(\xi^{\prime}, \eta^{\prime}, t^{\prime}\right) ; \eta^{\prime}=\eta, t^{\prime}=t\right\} .
\end{aligned}
$$

Any connected complete Riemannian manifold $M$ whose sectional curvatures lie between two constants can be written as $H / \Gamma$, where $H$ is a manifold as above, and $\Gamma$ is a subgroup of the isometry group of $H$, which can be identified with the fundamental group $\pi_{1}(M)$ of $M$. The action of $\Gamma$ extends naturally to the boundary $\partial H$. The elements of $\Gamma$ have one or two fixed points on $\partial H$; in the first case they are called parabolic, in the second case hyperbolic. An hyperbolic element $h$ fixes the geodesic joining its two fixed points. This geodesic projects on a closed geodesic in $M$ whose Frobenius element is equal to $h$, up to conjuguacy.

The limit set $\Lambda \Gamma$ of $\Gamma$ is the set of accumulation points in $\partial H$ of the orbit under $\Gamma$ of some point in $H$. It does not depend on the point chosen. The group $\Gamma$ is said to be elementary if this set has finitely many elements. This is equivalent to $\Gamma$ being virtually nilpotent.

The concept of Gibbs measure can be generalized to this setting [9], following a construction due to S.J. Patterson. These measures are supported by the non-wandering set of the flow. A finite Gibbs measure is ergodic; geometrically finite manifolds always admit finite Gibbs measures. We will only use some mild properties of the measure in the Hopf coordinates, which are explained below, so that we do not give details of the construction.

THEOREM 4. - Let $M$ be a connected complete Riemannian manifold $M$ whose sectional curvatures lie between two negative constants, and with nonelementary fundamental group. Let $\mu$ be a finite Gibbs measure on $S^{1} M$, and $\widehat{M}$ a connected regular cover of $M$ with nilpotent deck transformation group. Then the weak stable foliation $\widehat{W}^{\text {ws }}$ on $S^{1} \widehat{M}$ is ergodic with respect to $\mu \times$ Haar.

Moreover, if the lengths of the periodic geodesics on $\widehat{M}$ generate a dense subgroup of $\mathbb{R}$, then the horosphere foliation $\widehat{W}^{s s}$ is ergodic with respect to $\mu \times$ Haar.

REMARKS. - - Bowen-Margulis measure and harmonic measure are examples of Gibbs measures; they may be finite measures, depending on the geometry of M.

- The topological mixing of the flow $\widehat{\phi}_{t}$ on its non-wandering set in $\widehat{M}$ is equivalent to the condition $\overline{\langle\{\ell(\tau) ;[\tau]=0\}\rangle}=\mathbb{R}$, and to the transitivity of $\widehat{W}^{s s}$ on $\widehat{M}[12]$. It is known, for example, if there is a cusp on $\widehat{M}$, if $M$ is of finite volume, or if the curvature is constant [11]. It is conjectured to be always satisfied. 
- In constant curvature, the theorem can also be stated in term of group actions. The horospheres are the orbits of a unipotent subgroup of the isometry group of the manifold.

- The result can be generalized to $\mathrm{CAT}(-1)$ spaces. Finally, it is true if the deck transformation group is virtually nilpotent; one just applies the result with $M$ replaced by the finite extension over which $\widehat{M}$ is a nilpotent cover.

- In [16], U. Hamenstädt studied the ergodicity of $\widehat{W}^{w s}$ on $\widehat{M}$, when the manifold $M$ is compact; her approach is quite different from the one described here, and makes use of leafwise Laplacians, harmonic measures and diffusion processes. These methods seem difficult to generalize to the non-compact case. One problem comes from the fact that the harmonic measure may be infinite, non-ergodic, even on a finite volume manifold.

Proof. — The product structure may be defined globally on the universal cover $H$ of the manifold $M$, and then pushed down on the cover. The product of two points $\left(\xi_{1}, \eta_{1}, t_{1}\right)$ and $\left(\xi_{2}, \eta_{2}, t_{2}\right)$, in Hopf coordinates, is given by the formula

$$
\left\langle\left(\xi_{1}, \eta_{1}, t_{1}\right),\left(\xi_{2}, \eta_{2}, t_{2}\right)\right\rangle=\left(\xi_{2}, \eta_{1}, t_{2}\right)
$$

and belongs to $\widetilde{W^{s s}}\left(\widetilde{\phi}_{t_{2}-t_{1}}\left(\xi_{1}, \eta_{1}, t_{1}\right)\right) \cap \widetilde{W}^{s u}\left(\xi_{2}, \eta_{2}, t_{2}\right)$ as needed. This formula commutes with the action of the isometry group, so it is uniform in the fibers of the cover.

A Gibbs measure takes the following form in Hopf coordinates on $H$ :

$$
\mathrm{d} \mu(\xi, \eta, t)=\mathrm{e}^{\delta \beta_{x_{0}}(\xi, \eta)} \mathrm{d} \mu_{x_{0}}(\xi) \mathrm{d} \mu_{x_{0}}^{\prime}(\eta) \mathrm{d} t
$$

where $x_{0}$ is some point in $H$ chosen as origin. The function $\beta$ is bounded below and above on compact sets, by constants depending only on the distance from $x_{0}$ to the geodesic with endpoints $\xi$ and $\eta$; whereas the modulus of absolute continuity of $\mu_{x_{0}}$ with respect to some element $\gamma$ of the isometry group of $H$ is known, and bounded on $\partial H$ by a constant depending only on the distance from $\gamma x_{0}$ to $x_{0}$. We refer to [9] for an explicit formula, which is not needed here.

Let $\widetilde{v}$ be a periodic point on $H$ and $[\widetilde{v}$ the hyperbolic element which fixes the geodesic starting from $\widetilde{v}$ and for which $\phi_{\ell(\widetilde{v})}(\widetilde{v})=[\widetilde{v}] \widetilde{v}$. The transformation $T$ on $H$ which associates the point $\left\langle[\widetilde{v}]^{-1} \phi_{\ell(\widetilde{v})}(x), x\right\rangle$ to $x$ is given in the Hopf coordinates by the simple formula $(\xi, \eta, t) \mapsto\left(\xi,[\widetilde{v}]^{-1} \eta, t\right)$. Taking $x_{0}$ as the base point of the vector $v$, we see that the image of $\mu$ under $T$ is bounded by a constant times $\mu$ on balls centered on $x_{0}$, the constant depending only on the radius of the ball and on $\widetilde{v}$. Moreover the constant is the same for $\widetilde{v}$ and its images under the isometry group of $H$. This gives the estimate on the measure needed to apply Theorem 1.

It remains to check the condition on the periodic orbits.

BULletin DE LA SOCiÉtÉ MATHÉmATiQUe DE FRANCE 
LEMMA 5. - Let $\Gamma$ be a non-elementary subgroup of the group of isometry of a connected riemannian manifold with sectional curvatures lying between two negative constants. Then $\Gamma$ is generated by its hyperbolic elements.

Proof of Lemma 5. - One has to show that parabolic elements are products of hyperbolic elements. Let $p$ be a parabolic element and $p_{\infty}$ its fixed point in $\partial H$. The action of $p$ on $\Lambda \Gamma-\left\{p_{\infty}\right\}$ is free and properly discontinuous. So there is an open set $U \subset \Lambda \Gamma$, whose images under $p$ are disjoint. Let $V$ be an open ball whose closure is contained in $U$. The ends of periodic geodesics in $M$ are dense in $\Lambda \Gamma \times \Lambda \Gamma$ [14]. So, there is a hyperbolic element in $\Gamma$ whose fixed points are contained in $V$.

Taking some power of that element, we have found an hyperbolic element $h$ and two disjoint compact neighborhoods $V_{1}, V_{2} \subset V$ of the fixed points of $h$, which satisfy $h\left(V_{1}^{c}\right) \subset V_{2}$. The group $\langle p, h\rangle$ generated by $p$ and $h$ is called an extended Schottky group by F. Dal'bo and M. Peigné [13]. They show that it is a free group with generators $p$ and $h$. In particular the abelianized of $\langle p, h\rangle$ is equal to $\mathbb{Z} p \oplus \mathbb{Z} h$. It is moreover a geometrically finite group with one cusp, which implies that all parabolic elements are conjuguated to a power of $p$ [4]. So, the projection of a parabolic element in the abelianized group ends in the first factor $\mathbb{Z} p$. We deduce that the product of $p$ by $h$ is hyperbolic; this ends the proof of the lemma.

We return to the proof of the theorem.

There is a periodic orbit associated to each hyperbolic element in $\pi_{1}(M)$; the projections of hyperbolic elements of $\pi_{1}(M)$ in the deck transformation group of the cover can thus be realized as Frobenius elements of periodic geodesics. So, the lemma gives the equality $\left\langle\left\{[\tau] ;[\tau] \in G_{i}\right\}\right\rangle=G_{i} / G_{i-1}$, for any central series associated to the nilpotent group. If the flow is topologically mixing on the cover, we also have $\overline{\langle\{\ell(\tau) ;[\tau]=0\}\rangle}=\mathbb{R}$. In particular, $\mathbb{R} \times\{0\}$ belongs to the set $\overline{\left\langle\left\{(\ell(\tau),[\tau]) ;[\tau] \in G_{i}\right\}\right\rangle}$ for all $i$, hence

$$
\overline{\left\langle\left\{(\ell(\tau),[\tau]) ;[\tau] \in G_{i}\right\}\right\rangle}=\mathbb{R} \times G_{i} / G_{i-1} .
$$

This ends the proof.

Corollary 1. - Let $M$ be a connected finite volume manifold whose sectional curvatures lie between two negative constants, and with first partial derivatives of the curvatures bounded on $M$. Let $\widehat{M}$ be a connected nilpotent cover of $M$. Then the strong stable foliation on $\widehat{M}$ is ergodic with respect to the Liouville measure.

REMARK. - On a compact manifold, the absolute continuity of the Liouville measure with respect to the foliations is due to D.V. Anosov [2]. The potential $f(x)=-\mathrm{d} / \mathrm{d} t_{\mid t=0}\left(\ln \left|\operatorname{det}\left(D_{x} \phi_{t_{\mid T_{x} W^{s u}}}\right)\right|\right)$, associated to the Liouville measure, is Hölder continuous, so that it is a Gibbs measure. The proof extends

TOME $131-2003-\mathrm{N}^{\mathrm{O}} 2$ 

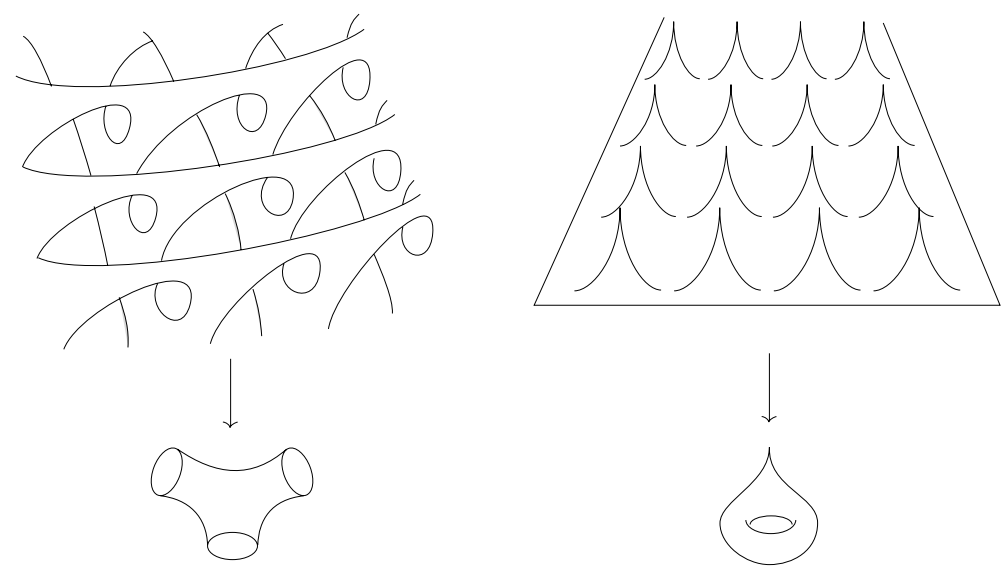

FiguRE 2. Left: Thick part of the thrice punctured sphere. Right: Punctured torus

straightforwardly to the finite volume case if the first derivatives of the curvatures are bounded; this remark has been made by several authors, for example M. Brin [8].

EXAmples: HOMOLOGY COVERS. — The corollary may be applied to homology covers. The thick part of the thrice punctured sphere is on the left of the picture; the once punctured torus is on the right. These are finite volume manifolds and their horospheric foliations are ergodic on the cover with respect to the Liouville measure.

Acknowledgements. - I wish to thank F. Ledrappier and L. Flaminio for their help and comments.

\section{BIBLIOGRAPHY}

[1] Aaronson (J.), Solomyak (R.) \& Sarig (O.) - Tail-invariant measures for some suspension semiflows, to appear.

[2] Anosov (D.V.) - Geodesic flows on closed riemannian manifolds with negative curvature, Proc. Steklov Inst. Math., vol. 90, American Mathematical Society, Providence, R.I., 1967, English translation 1969.

[3] Babillot (M.) \& Ledrappier (F.) - Geodesic paths and horocycle flow on Abelian covers, in Proceedings of the International Colloquium on Lie groups and ergodic theory (Mumbai 1996), Narosa Publishing House, NewDehli, 1998, pp. 1-32. 
[4] Bowditch (B.H.) - Geometrical finiteness with variable curvature, Duke Math. J., t. 77 (1995), no. 1, pp. 229-274.

[5] Bowen (R.) - Periodic orbits for hyperbolic flows, Amer. J. Math., t. 94 (1972), pp. 1-30.

[6] Bowen (R.) \& Marcus (B.) - Unique ergodicity for horocycle foliations, Israel J. Math., t. 13 (1977), pp. 43-67.

[7] Bowen (R.) \& Ruelle (D.) - The ergodic theory of Axiom A flows, Invent. Math., t. 29 (1975), pp. 153-170.

[8] BRIN (M.) - Ergodicity of the geodesic flow, Notes from Mathematical Research Summer Institute, Seattle, 1999.

[9] Coudene (Y.) - Gibbs measures on negatively curved manifolds, to appear.

[10] Cocycles and stable foliations of axiom a flows, Ergodic Theory Dynamical Systems, t. 21 (2001), pp. 767-775.

[11] DaL'Bo (F.) - Remarques sur le spectre des longueurs d'une surface et comptages, Bol. Soc. Brasil. Mat. (new series), t. 30 (1999), no. 2, pp. 199 221.

[12] _ Topologie du feuilletage fortement stable, Ann. Inst. Fourier (Grenoble), t. 50 (2000), no. 3, pp. 981-993.

[13] Dal'bo (F.) \& Peigné (M.) - Some negatively curved manifolds with cusps, mixing and counting, J. reine angew. Math., t. 497 (1998), pp. 141169.

[14] EBerlein (P.) - Geodesic flows on negatively curved manifolds I, Ann. of Math. (2), t. 95 (1972), pp. 492-510.

[15] Furstenberg (H.) - The unique ergodicity of the horocycle flow, in Recent advances in topological dynamics, Lect. Notes in Math., vol.318, Springer, 1973, pp. 95-115.

[16] Hamenstädt (U.) - Ergodic properties of gibbs measures on nilpotent covers, to appear.

[17] Hedlund (G.A.) - Fuchsian groups and transitive horocycles, Duke Math. J., t. 2 (1936), pp. 530-542.

[18] _ Fuchsian groups and mixtures, Ann. of Math., t. 40 (1939), pp. 370-383.

[19] Hopf (E.) - Fuchsian groups and ergodic theory, Trans. Amer. Math. Soc., t. 39 (1936), pp. 299-314.

[20] Kaimanovich (V.) - Ergodic properties of the horocycle flow and classification of Fuchsian groups, J. Dynam. Control Sys., t. 6 (2000), no. 1, pp. 21-56.

[21] Kaimanovich (V.) \& Schmidt (K.) - Ergodicity of cocycles 1: general theory, to appear.

[22] Livsic (A.) - Cohomology of dynamical systems, Izv. Akad. Nauk SSSR Ser. Mat., t. 6 (1972), pp. 1278-1301.

TOME $131-2003-\mathrm{N}^{\mathrm{O}} 2$ 
[23] Pollicott (M.) - $\mathbb{Z}^{d}$ covers of horosphere foliations, Discrete Cont. Dynam. Sys., t. 6 (2000), no. 1, pp. 147-154.

[24] Ruelle (D.) - Thermodynamical formalism, Encyclopedia of Mathematics and its applications, vol.5, Addison-Wesley Publishing Compagny, Reading MA, 1978.

[25] Shub (M.) - Global stability of dynamical systems; with the collaboration of A. Fathi, R. Langevin, Springer Verlag, New York, etc., 1987, Transl. from French by J. Christy.

[26] Smale (S.) - Differentiable dynamical systems, Bull. Amer. Math. Soc. (N.S.), t. 73 (1967), pp. 747-817.

[27] Solomyak (R.) - A short proof of ergodicity of Babillot-Ledrappier measures, Proc. Amer. Math. Soc., t. 129 (2001), no. 12, pp. 3589-3591. 